\title{
CONSISTENCY CONDITIONS FOR DIMER MODELS
}

\author{
RAF BOCKLANDT \\ School of Mathematics and Statistics, Herschel Building, \\ Newcastle University, Newcastle upon Tyne, NE1 7RU, UK \\ e-mail: raf.bocklandt@gmail.com
}

(Received 23 November 2010; revised 29 April 2011; accepted 3 August 2011)

\begin{abstract}
Dimer models are a combinatorial tool to describe certain algebras that appear as noncommutative crepant resolutions of toric Gorenstein singularities. Unfortunately, not every dimer model gives rise to a noncommutative crepant resolution. Several notions of consistency have been introduced to deal with this problem. In this paper, we study the major different notions in detail and show that for dimer models on a torus, they are all equivalent.
\end{abstract}

2010 Mathematics Subject Classification. 14M25, 14A22, 16S38.

1. Introduction. If $X$ is a three-dimensional normal Gorenstein singularity admitting a crepant resolution $\tilde{X} \rightarrow X$, then one is interested to describe the bounded derived category $\mathcal{D}$ Coh $\tilde{X}$ of coherent sheaves on $\tilde{X}$. A well-known result by Bridgeland [4] shows that this category only depends on the singularity and not on the particular choice of crepant resolution.

In many cases, there exists a tilting bundle in $\mathcal{U} \in \mathcal{D} \operatorname{Coh} \tilde{X}$ such that $\mathcal{D} \operatorname{Coh} \tilde{X}$ is equivalent as a triangulated category to the derived category of finitely generated $A$-modules $\mathcal{D} \operatorname{Mod} A$, where $A=\operatorname{End} \mathcal{X}$. To model these algebras without referring to a commutative crepant resolution, Van den Bergh [23] introduced the notion of a noncommutative crepant resolution (NCCR) of $X$. This is a homologically homogeneous algebra of the form $A=\operatorname{End}_{R}(T)$, where $T$ is a reflexive $R$-module, with $R=\mathbb{C}[X]$ the coordinate ring of the singularity. An NCCR is, however, far from unique and in general there are an infinite number of different noncommutative crepant resolutions.

If we make two restrictions, the problem becomes more manageable. First, we assume that $X$ is a toric three-dimensional Gorenstein singularity. This automaticly implies the existence of a commutative crepant resolution. Secondly, we assume that the tilting bundle is a direct sum of nonisomorphic line bundles. It was noticed in string theory $[\mathbf{8}, \mathbf{1 0}, \mathbf{1 1}]$ that, under these conditions, the algebra $A$ can be described using a dimer model on a torus.

This means that $A$ is the path algebra of a quiver $Q$ with relations where $Q$ is embedded in a two-dimensional real torus $T$ such that every connected piece of $T \backslash Q$ is bounded by a cyclic path of length at least 3 . The relations are given by demanding for every arrow $a$ that $p=q$ where $a p$ and $a q$ are the bounding cycles that contain $a$.

This nice description follows from the fact that the algebra $A$ is a toric order, a special type of order compatible with the toric structure, and Calabi-Yau-3 (CY-3), i.e. it admits a self-dual bimodule resolution of length 3. In [2], it was shown that every toric CY-3 order comes from a dimer model.

Not every dimer model gives a noncommutative crepant resolution of its centre. To do so, it needs to satisfy some extra conditions called consistency conditions. In recent years, 
several quite different consistency conditions were proposed such as cancellation [7], nonintersecting zig and zag rays [16], consistent R-charges [15] and algebraic consistency $[5]$.

The aim of this paper is to show that for dimer models on a torus, all these consistency conditions are equivalent. Moreover, the condition of being an order and the condition of being an NCCR are also equivalent to these consistency conditions. The situation for the Calabi-Yau condition is less clear: it was shown by Davison [7] and Broomhead [5] that cancellation and algebraic consistency imply the CY-3 condition, but there is no proof for the other direction. We will give an example of an infinite dimer model that is not cancellation but satisfies a suitable generalization of the CY-3 property to the infinite case. However, no finite counterexamples are known.

If one broadens the definition of a dimer model to allow other compact surfaces, the consistency conditions are no longer equivalent. We will also discuss the differences for those cases.

The paper is organized as follows. We start with a preliminary section on quivers and dimer models. Then we introduce the cancellation property and discuss its relation with the CY-3 property. In the other sections, we introduce each time a notion of consistency and show that it is equivalent to the cancellation property for dimer models on a torus. We end with a summary section which gives an overview of which equivalences hold for each type of surface.

\section{Preliminaries.}

2.1. Quivers. A quiver $Q$ is an oriented graph. We denote the set of vertices by $Q_{0}$, the set of arrows by $Q_{1}$ and the maps $h, t$ assign to each arrow its head and tail. A nontrivial path $p$ is a sequence of arrows $a_{1} \cdots a_{k}$ such that $t\left(a_{i}\right)=h\left(a_{i+1}\right)$, whereas a trivial path is just a vertex. We will denote the length of a path by $|p|:=k$ and the head and tail by $h(p)=h\left(a_{1}\right), t(p)=t\left(a_{k}\right)$. A path is called cyclic if $h(p)=t(p)$. Later on we will denote by $p[i]$ the $n-i$ th arrow of $p$ and by $p[i \ldots j]$ the subpath $p[i] \ldots p[j]$.

$$
O \longleftarrow^{p[n-1]} \bigcirc \overbrace{}^{p[n-2]} O<^{p[1]} O \leftarrow^{p[0]} \bigcirc \text { and } p=p[n-1] p[n-2] \ldots p[1] p[0] .
$$

A quiver is called connected if it is not the disjoint union of two subquivers and it is strongly connected if there is a cyclic path through each pair of vertices.

The path algebra $\mathbb{C} Q$ is the complex vector space with as basis the paths in $Q$ and the multiplication of two paths $p, q$ is their concatenation $p q$ if $t(p)=h(q)$ or else 0 . The span of all paths of nonzero length forms an ideal which we denote by $\mathcal{J}$. A path algebra with relations $A=\mathbb{C} Q / \mathcal{I}$ is the quotient of a path algebra by a finitely generated ideal $\mathcal{I} \subset \mathcal{J}^{2}$. A path algebra is connected or strongly connected if and only if its underlying quiver is. We will call a path algebra with relations $\mathbb{C} Q / \mathcal{I}$ positively graded if there exists a grading $\mathrm{R}: Q_{1} \rightarrow \mathbb{R}_{>0}$ such that $\mathcal{I}$ is generated by homogeneous relations.

2.2. Dimer models. A dimer model $\mathcal{Q}$ is a strongly connected quiver $Q$ enriched with two disjoint sets of cycles of length at least 3: $Q_{2}^{+}$and $Q_{2}^{-}$, such that

DO Orientability condition. Every arrow is contained exactly once in one cycle in $Q_{2}^{+}$and once in one in $Q_{2}^{-}$. 
DM Manifold condition. The incidence graph of the cycles and arrows meeting a given vertex is connected.

The Euler characteristic of a dimer model is

$$
\chi_{\mathcal{Q}}:=\# Q_{0}-\# Q_{1}+\# Q_{2} \text { with } Q_{2}=Q_{2}^{+} \cup Q_{2}^{-} .
$$

From a dimer model, we can build a topological space $|\mathcal{Q}|$ by associating with every cycle of length $k$ in $Q_{2}$ a $k$-gon. We label the edges of this $k$-gon cyclicly by the arrows of the quiver and identify edges of different polygons labelled with the same arrow. If $\mathcal{Q}$ satisfies (DO) and (DM), then $|\mathcal{Q}|$ is a compact orientable surface with Euler characteristic $\chi_{\mathcal{Q}}$ ([2]), such that the cycles in $Q_{2}^{+}$will be oriented anticlockwise and those in $Q_{2}^{-}$clockwise. We say that $\mathcal{Q}$ is a dimer model on $|\mathcal{Q}|$ and if $\chi_{\mathcal{Q}}=0$, we speak of a dimer model on a torus.

To every dimer model, we can associate its Jacobi algebra. For every $a \in Q_{1}$, we set $r_{a}=p^{+}-p^{-}$, where $p^{ \pm} a \in Q_{2}^{ \pm}$, and then set

$$
A_{\mathcal{Q}}:=\mathbb{C} Q /\left\langle r_{a} \mid a \in Q_{1}\right\rangle
$$

This algebra can be expressed in terms of a superpotential but we will not pursue this direction.

A dimer model is positively graded if there is a degree map $\mathrm{R}: Q_{1} \rightarrow \mathbb{R}_{>0}$ such that all cycles in $Q_{2}$ have the same degree. This turns $A_{\mathcal{Q}}$ in a positively graded algebra.

Given a dimer model $\mathcal{Q}$ on $X=|\mathcal{Q}|$, we can look at the universal cover $\tilde{X} \rightarrow X$. We can lift the embedding of $\mathcal{Q}$ in $X$ to obtain a possible infinite dimer model $\tilde{\mathcal{Q}}$, which we will call the universal cover of $\mathcal{Q}$.

REMARK 2.1. Usually, the definition of a dimer model starts from the dual of $\mathcal{Q}$ : a bipartite graph on a surface, with nodes $Q_{2}$ and edges $Q_{1}$, only after that the quiver is constructed by taking the dual. We do not do this because the switching can sometimes cause confusion. We do keep the name dimer model, as it is most commonly used in the literature.

EXAMPLE 2.2. Below, we give four examples of dimer models: the first is a dimer model on a sphere, the second and the third on a torus, and the last on a double torus. Arrows and vertices with the same label are identified.
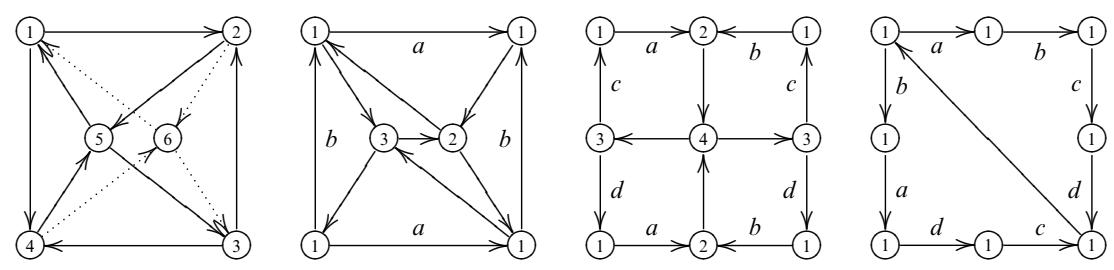

In the first example, the cycles of $Q_{2}^{-}\left(Q_{2}^{+}\right)$are the (anti-)clockwise triangles of the octahedron, and in the last three examples, the cycles $Q_{2}^{-}\left(Q_{2}^{+}\right)$are (anti-)clockwise triangles, quadrangles and pentagons.

Using the results of this paper, one can check that the first example is an order but not CY-3, the second neither an order nor CY-3, the third both an order and CY-3 and the last CY-3 but not an order. The third is the only NCCR and its centre is the coordinate ring over the cone over $\mathbb{P}_{1} \times \mathbb{P}_{1}$. 
3. Cancellation. A path algebra of a quiver with relations is called a cancellation algebra if for every arrow $a$ and any two paths $p, q$ with $h(a)=t(p)=t(q)$, we have $p a=$ $q a \Longrightarrow p=q$ and for any two paths $p, q$ with $t(a)=h(p)=h(q)$, we have $a p=a q \Longrightarrow$ $p=q$.

For Jacobi algebras from dimer models, we can restate the cancellation property. The relations in the Jacobi algebra $A_{\mathcal{Q}}$ imply that all cycles in $Q_{2}$ are equivalent: $c_{1} p=p c_{2}$ for every $p$ with $h(p)=t\left(c_{1}\right)$ and $t(p)=h\left(c_{2}\right)$. This implies that the algebra $A_{\mathcal{Q}}$ has a central element: $\sum c$ where we sum over a subset representative of $Q_{2}$ that contains just one cyclic path $c$ with $h(c)=i$ for every $i \in Q_{0}$. We will denote this central element by $\ell$. For every arrow $a$, we can find a path $p$ such that $a p \in Q_{2}^{+}$and hence $a p=h(a) \ell$ and $p a=t(a) \ell$.

The cancellation property states that the map

$$
A_{\mathcal{Q}} \rightarrow A_{\mathcal{Q}} \otimes_{\mathbb{C}[\ell]} \mathbb{C}\left[\ell, \ell^{-1}\right]
$$

is an embedding. This tensor product is the algebra obtained by making every arrow invertible (i.e. for every $a$, we have an $a^{-1}$ such that $a a^{-1}=h(a)$ and $a^{-1} a=t(a)$ ). This algebra is the localization of $A_{\mathcal{Q}}$ by the Ore set $\left\{\ell^{k} \mid k \in n\right\}$ and we denote it by $\hat{A}_{\mathcal{Q}}$.

We will take this property as the starting point from which we are going to prove the equivalences of the different consistency conditions. But first we need to discuss the relation between cancellation and the Calabi-Yau property.

4. The Calabi-Yau-3 Condition. Definition 4.1. A path algebra with relations $A$ is Calabi-Yau-3 (CY-3) if $A$ has a projective bimodule resolution $\mathcal{P}^{\bullet}$ that is dual to its third shift

$$
\mathrm{RHom}_{A-A}\left(\mathcal{P}^{\bullet}, A \otimes A\right)[3] \cong \mathcal{P}^{\bullet}
$$

Note that $A \otimes A$ has two-bimodule structures: the outer and the inner. The outer one is used to define what a homomorphism to $A \otimes A$ is and the inner one to turn $\mathrm{RHom}_{A-A}\left(\mathcal{P}^{\bullet}, A \otimes\right.$ A)[3] into a complex of $A$-bimodules.

From this definition, it is clear that a CY-3 algebra has global dimension 3 and there are isomorphism between $\operatorname{Ext}^{i}(X, Y)$ and $\operatorname{Ext}^{3-i}(X, Y)^{*}$ for every pair of finite-dimensional left $A$-modules $X, Y$. For more information about the CY-3 property, we refer to and [9] [1].

That cancellation implies CY-3 was proved by Ben Davison in [7].

THEOREM 4.2 (Davison). The Jacobi algebra of a dimer model $\mathcal{Q}$ with $\chi_{\mathcal{Q}} \leq 0$ is $C Y-3$ if it is a cancellation algebra.

REMARK 4.3. Davison's work was a generalization of work by Mozgovoy and Reineke [19], which used an extra consistency condition. This extra condition turned out to be a consequence of the cancellation property.

The method in the proof involved showing that a certain complex $\mathcal{C}^{\bullet}$, which is by construction dual to its third shift, is acyclic. This complex looks like

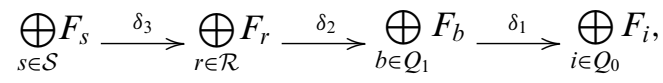


where $\mathcal{R}=\left\{r_{a} \mid a \in Q_{1}\right\}$ is the set of relations and $\mathcal{S}=\left\{v \ell \mid v \in Q_{0}\right\}$. The bimodule $F_{p}$ is defined as $A h(p) \otimes p \otimes t(p) A$. The differentials have the following form:

$$
\begin{aligned}
& \delta_{1}\left(q_{1} \otimes b \otimes q_{2}\right)=q_{1} b \otimes t(b) \otimes q_{2}-q_{1} \otimes h(b) \otimes b q_{2}, \\
& \delta_{2}\left(q_{1} \otimes r \otimes q_{2}\right)=\sum_{k} q_{1} a_{1} \ldots \otimes a_{k} \otimes \ldots a_{n} q_{2}-\sum_{k} q_{1} b_{1} \ldots \otimes b_{k} \otimes \ldots b_{m} q_{2}, \\
& \delta_{3}\left(q_{1} \otimes s \otimes q_{2}\right)=\sum_{h(a)=h(s)} q_{1} a \otimes r_{a} \otimes 1-\sum_{t(a)=h(s)} 1 \otimes r_{a} \otimes a q_{2} .
\end{aligned}
$$

In the second line, we assume that $r=a_{1} \ldots a_{n}-b_{1} \ldots b_{m}$.

Let $G$ be the groupoid of paths in $\hat{A}_{\mathcal{Q}}$, this groupoid gives a $G$-grading on every $F_{p}$ and this grading makes the complex homogeneous (see [7]).

This complex can even be defined for infinite dimer models, but in that case, the complex is not dual to its third shift because taking the dual is not well-behaved for infinite direct sums. We will still continue to call $A_{\mathcal{Q}} \mathrm{CY}-3$ in the infinite case if $\mathcal{C}$ is exact.

With this in mind, we have the following observation:

OBSERVATION 4.4. There are infinite dimer models that are CY-3 but not cancellation.

Proof. (Sketch). The example we give is

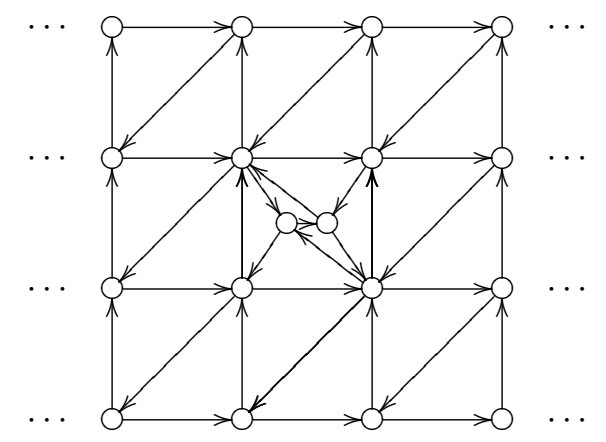

with all squares alike except the central one. We can give all arrows degree 1 to make $A_{\mathcal{Q}}$ graded.

If $\mathcal{C}^{\bullet}$ were not a bimodule resolution, then there would be extra syzygies. Because of the gradedness, there would be at least one simple $S_{v}:=A_{\mathcal{Q}} v /\left(A_{\mathcal{Q}} v\right)_{>0}$ for which $\mathcal{C} \bullet \otimes_{A_{\mathcal{Q}}}$ $S_{v}$ is not a projective resolution of $v$.

Now $\mathcal{C}^{\bullet} \otimes_{A_{\mathcal{Q}}} S_{v}$ can be decomposed in its $G$-homogeneous components and for each of the paths $p$ in $G$, one can check easily (see [3]) that the $p$-homogeneous part $\mathcal{C}^{\bullet} \otimes_{A_{\mathcal{Q}}} S_{v}$ is finite dimensional and acyclic.

It is still an open question whether in the finite case, there are CY-3 algebras that are not cancellation. 
5. Zigzag paths. Checking whether $A_{\mathcal{Q}}$ is a cancellation algebra is not an easy task. Here, we will introduce a combinatorial criterion that will enable us to check this property visually. In order to do this, we need the following theorem from [2] relating a dimer model and its universal cover.

THEOREM 5.1. The Jacobi algebra $A_{\mathcal{Q}}$ is a cancellation algebra if and only if the Jacobi algebra of the universal cover $A_{\tilde{\mathcal{Q}}}$ is a cancellation algebra.

We will now restrict ourselves to the case when the Euler characteristic is nonpositive, so from now on, we can assume that $|\tilde{\mathcal{Q}}|$ is a contractible manifold. This makes it possible to define the interior of a closed curve. This assumption allows us to deduce the following lemma:

Lemma 5.2. If $|\tilde{\mathcal{Q}}|$ is contractible, then $p \neq p \ell^{k}$ in $A_{\tilde{\mathcal{Q}}}$ for any path $p$ and $k>0$.

Proof. Identify $|\tilde{\mathcal{Q}}|$ with the complex plane and construct an index to the cycles in $\mathcal{Q}_{2}$ such that the positive cycles have even numbers and the negative odd. Let $z_{i}$ be the centre of the ith cycle.

If $p, q$ are the paths with the same head and tail, we can define the following invariant:

$$
I(p, q)=\lim _{N \rightarrow \infty} \oint_{p q^{-1}} \sum_{i=1}^{N} \frac{(-1)^{i}}{z_{i}} .
$$

It is clear that this is well-defined as the integral stabilizes for $N \rightarrow \infty$ : adding poles outside $p q^{-1}$ does not change it. If one applies a relation to $p$ or $q$, one positive pole and one negative will change sides. The signs in the poles keep the integral invariant. The lemma follows from the fact that $I\left(p \ell^{k}, p\right)=2 k \pi i$.

We can split any given path $p$ in the universal cover into positive (negative) arcs. These are maximal subpaths that are contained in a positive (negative) cycle. We will call a path positively (negatively) irreducible if none of its positive (negative) arcs contains $p^{+}\left(p^{-}\right)$for some defining relation $r_{a}=p^{+}-p^{-}$. A pair of paths $(p, q)$ with $h(p)=h(q)$ and $t(p)=h(q)$ such that $p q^{-1}$ is an anticlockwise loop that does not self-intersect and $p$ is positively irreducible and $q$ negatively irreducible is called an irreducible pair. The interior of an irreducible pair (i.e. the interior of the loop $p q^{-1}$ ) cannot be shrunk using the defining relations.

LEMMA 5.3. If $|\tilde{\mathcal{Q}}|$ is contractible, then $A_{\tilde{\mathcal{Q}}}$ is a cancellation algebra if and only if it has no irreducible pair.

Proof. The condition is necessary because an irreducible pair $(p, q)$ implies that in $\hat{A}_{\tilde{Q}}$, either $p=q \ell^{k}$ or $q=p \ell^{k}$ holds for some $k \geq 0$ but does not hold in $A_{\tilde{\mathcal{Q}}}$, so the map $A_{\tilde{\mathcal{Q}}} \rightarrow \hat{A}_{\tilde{\mathcal{Q}}}$ is not an injection.

To prove sufficiency, we suppose that there are no irreducible pairs. If $(p, q)$ is a pair of paths such that $p q^{-1}$ is a non-self-intersecting anticlockwise loop, then $p=q \ell^{k}$ or $q=p \ell^{k}$ for some $k \geq 0$. If not, such a pair $(p, q)$ for which $p q^{-1}$ has the least number of cycles in its interior forms an irreducible pair (a reduction would give us a pair with a smaller number of cycles in its interior). In particular, this shows that every loop can be reduced to a power of $\ell$ (take for $q$ a path of length 0 ).

Now, take two nonequivalent paths $p, q$ with the same head and tail such that $p \ell^{k}=$ $q \ell^{k}$ for some $k>0$. To prove cancellation, we must show that $p=q$. 
As already mentioned, we can turn every loop in $p$ or $q$ into a power of $\ell$. After puting these powers in front, we can split $p=\ell^{u} p_{1} \ldots p_{k}$ and $q=\ell^{v} q_{1} \ldots q_{l}$ such that $p_{i}$ and $q_{i}$ coincide or do not intersect. For each pair $\left(p_{i}, q_{i}\right)$, we have that $p_{i}=q_{i} \ell^{k_{i}}$ or $q_{i}=p_{i} \ell^{k_{i}}$ for some $k_{i} \geq 0$.

The sum of $u$ and the $k_{i}$ for which the first equality holds must equal the sum of $v$ and the $k_{i}$ for which the second equality holds. Otherwise $p \ell^{l}=q \ell^{l^{\prime}}$ for $l^{\prime} \neq l$, which is forbidden by Lemma 5.2 as we supposed that $p \ell^{k}=q \ell^{k}$.

To turn $p$ into $q$, we first do the transformations $p_{i} \rightarrow q_{i} \ell^{k_{i}}$, then we distribute the powers $\ell$ over the pieces of $p$ we have not transformed yet and then we apply transformations $p_{i} \ell^{k_{i}} \rightarrow p_{i}$.

REMARK 5.4. We used the notation $\ell$ although in the universal cover $\ell$ is an infinite sum and hence not an element of $A_{\tilde{\mathcal{Q}}}$. The products $\ell p$ and $p \ell$ are still defined because they single out one term of $\ell$.

A zigzag path is an infinite length path $\mathcal{Z}=\ldots \mathcal{Z}[2] \mathcal{Z}[1] \mathcal{Z}[0] \mathcal{Z}[-1] \mathcal{Z}[-2] \ldots$ for which all positive and negative arcs have length 2 . It is easy to see that there are exactly two zigzag paths for which $\mathcal{Z}[0]$ equals a given arrow $a(\mathcal{Z}[1] \mathcal{Z}[0]$ is either a positive or a negative arc). We denote these two zigzag paths by $\mathcal{Z}_{a}^{+}$and $\mathcal{Z}_{a}^{-}$. The part of the zigzag path $\mathcal{Z}_{a}^{+}$starting from $a, \prod_{i \geq 0} \mathcal{Z}_{a}^{+}[i]$ is called the zig ray from $a$ and is denoted by $\overrightarrow{\mathcal{Z}}_{a}^{+}$. Similarly, we denote the zag ray by $\overrightarrow{\mathcal{Z}}_{a}^{-}$. The notion of a zigzag path is based on work by Kenyon in [16] and Kenyon and Schlenker in [17].

Every zigzag path $\mathcal{Z}$ is bounded by a positively and a negatively irreducible path consisting of the positive (negative) arcs $u_{i}$ such that $u_{i} \mathcal{Z}[2 i+1] \mathcal{Z}[2 i]$ is a positive (negative) cycle.

THEOREM 5.5. If $\chi_{\mathcal{Q}} \leq 0$, then $\mathcal{Q}$ is cancellation if and only iffor every arrow $a \in \tilde{Q}_{1}$, the following condition hold:

$\mathrm{Z} \overrightarrow{\mathcal{Z}}_{a}^{+}$and $\overrightarrow{\mathcal{Z}}_{a}^{-}$only intersect in a, i.e.

$$
\forall i, j>0: \mathcal{Z}_{a}^{+}[i] \neq \mathcal{Z}_{a}^{-}[j]
$$

(note that the zigzag paths can intersect but only in different directions $\left(f . i . \mathcal{Z}_{a}^{+}[i]=\right.$ $\mathcal{Z}_{a}^{-}[j]$ with $i>0$ and $\left.j<0\right)$.

REMARK 5.6. Condition ( $Z$ ) also implies that a zigzag path cannot self-intersect. Indeed if there are self-intersecting zigzag paths, we can take $\mathcal{Z}$ such that $a=\mathcal{Z}[0]=\mathcal{Z}[i]$ and the loop $\mathcal{Z}[i-1] \ldots \mathcal{Z}[0]$ encompasses the smallest number of cycles. If $\mathcal{Z}=\mathcal{Z}_{a}^{ \pm}$, then the zigzag path $\mathcal{Z}_{a}^{\mp}$ has an arrow inside the loop and as it cannot make a smaller loop, it must enter and leave the loop, and hence there the zig and the zag ray of some arrow in $\mathcal{Z}$ intersect.

\section{Proof of Theorem 5.5. Condition $(\mathbf{Z})$ is necessary.}

If $\mathcal{Z}_{a}^{+}[i]=\mathcal{Z}_{a}^{-}[j]$ and $i, j$ are both positive and minimal, we look at the paths $p_{+}, p_{-}$that are the irreducible paths accompanying $\mathcal{Z}_{a}^{+}[i] \ldots \mathcal{Z}_{a}^{+}[0]$ and $\mathcal{Z}_{a}^{-}[j] \ldots \mathcal{Z}_{a}^{-}[0]$ which lie in the exterior of $\mathcal{Z}_{a}^{+}[i] \ldots \mathcal{Z}_{a}^{+}[0]\left(\mathcal{Z}_{a}^{-}[j] \ldots \mathcal{Z}_{a}^{-}[0]\right)^{-1}$. By construction, these paths form an 
irreducible pair.

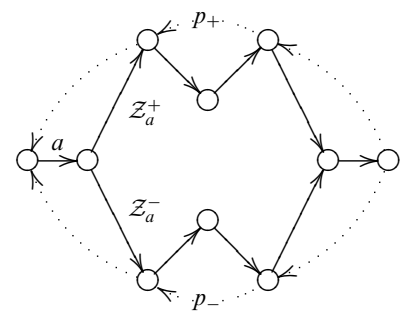

\section{Condition $(Z)$ is sufficient.}

If $\mathcal{Q}$ is not cancellation, we will assume that condition $(Z)$ holds and search for a contradiction. By Lemma 5.3, let $(p, q)$ be an irreducible pair and let $S$ be the interior of $p q^{-1}$.

Let $a_{0}$ be the last arrow of $p$ and consider the zigzag path $\mathcal{Z}_{1}=\mathcal{Z}_{a_{0}}^{+}$. By construction, $\mathcal{Z}_{1}[1]$ sits inside $S$, and because $S$ only contains a finite number of arrows, there must be a minimal $j>0$ such that $\mathcal{Z}_{1}[j]$ does not sit in $S$. The arrow $\mathcal{Z}_{1}[j]$ must sit on the boundary of $S$, so it must either be an arrow of $p$ or $q$.

- If $\mathcal{Z}_{1}[j]$ is an arrow of $p$, then $j$ must be odd because $p$ bounds only positive cycles from $S$. The zigzag path $\mathcal{Z}_{1}$ and a part $p^{(1)}$ of $p$ cut out a piece of $S$, which we will denote by $S^{(1)}$. Now put $a_{1}=\mathcal{Z}_{1}[j-1]$ and look at a zigzag path $\mathcal{Z}_{2}=\mathcal{Z}_{a_{1}}^{-}$. Because $j$ is odd, this zigzag path comes from the inside of $S^{(1)}$. Condition $(Z)$ implies that $\mathcal{Z}_{2}$ cannot intersect $\mathcal{Z}_{1}$ twice, so it must have entered $S^{(1)}$ somewhere on $p^{(1)}$ but not at $a_{0}$.

The zigzag path $\mathcal{Z}_{2}$ and a strictly smaller path $p^{(2)} \subset p^{(1)}$ cut out a piece of $S^{(1)}$, which we will denote by $S^{(2)}$. Now let $a_{2}$ be the first arrow on $\mathcal{Z}_{2}$ inside $S^{(1)}$ and look at a zigzag path $\mathcal{Z}_{3}=\mathcal{Z}_{a_{2}}^{+}$. $\mathcal{Z}_{3}$ cannot intersect $\mathcal{Z}_{2}$ twice, so $\mathcal{Z}_{3}$ must leave $S^{(2)}$ somewhere along the path $p^{(2)}$ but not in the first arrow.

The zigzag path $\mathcal{Z}_{3}$ and a strictly smaller path $p^{(3)} \subset p^{(2)}$ cut out a piece of $S^{(2)}$. Proceeding like this, we get a sequence of shrinking pieces until $p^{(i)}$ has length zero. But this implies that the corresponding zigzag path $\mathcal{Z}_{i}$ will self-intersect (contradicting Remark 5.6).

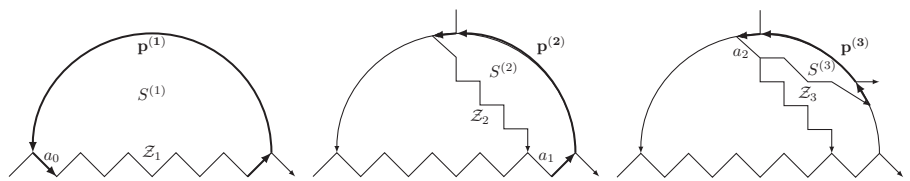

- If $\mathcal{Z}_{1}[j]$ is an arrow of $q$, then $j$ must be even and we can copy the argument above by changing the $p$ in $q$.

REMARK 5.7. Dimer models with positive Euler characteristic can never satisfy condition $(Z)$ because the universal cover is the quiver itself. As this quiver is finite, the zig and zag rays intersect multiple times.

REMARK 5.8. A similar discussion on the relation between cancellation and zigzag paths was done by Ishii and Ueda in $[13,14]$

6. Consistent R-Charges. We borrow the following definition from Kennaway [15]:

Definition 6.1. A grading $\mathrm{R}: Q_{1} \rightarrow \mathbb{R}_{>0}$ is a consistent $\mathrm{R}$-charge if 
$\mathrm{R} 1 \forall c \in Q_{2}: \sum_{a \in c} \mathrm{R}_{a}=2$,

$\mathrm{R} 2 \forall v \in Q_{0}: \sum_{h(a)=v}\left(1-\mathrm{R}_{a}\right)+\sum_{t(a)=v}\left(1-\mathrm{R}_{a}\right)=2$.

REMARK 6.2. In [5], a distinction is made between geometrically consistent and marginally consistent $\mathrm{R}$-charges. The former have the extra condition that $\mathrm{R}_{a}<1$ for every $a$, while for the latter, one also allows $\mathrm{R}_{a} \geq 1$. We will not make this distinction: for us marginally consistent $\mathrm{R}$-charges are also consistent.

It has been pointed out in e.g. [15] that consistency implies that the Euler characteristic is zero

$$
\begin{aligned}
2 \chi_{\mathcal{Q}} & =2\left(\# Q_{2}-\# Q_{1}+\# Q_{0}\right) \\
& =\sum_{c \in Q_{2}} \sum_{a \in c} \mathrm{R}_{a}-\sum_{a} 2+\sum_{v}\left(\sum_{h(a)=v}\left(1-\mathrm{R}_{a}\right)+\sum_{t(a)=v}\left(1-\mathrm{R}_{a}\right)\right) \\
& =\sum_{a}(\underbrace{2 \mathrm{R}_{a}}_{a \text { sits in } 2 \text { cycles }}-2+\underbrace{1-\mathrm{R}_{a}}_{v=h(a)}+\underbrace{1-\mathrm{R}_{a}}_{v=t(a)})=0 .
\end{aligned}
$$

Given a consistent R-charge, we can realize the universal cover of the dimer model, which is the Euclidean plane, in the following way: turn every cycle in $Q_{2}$ into a polygon the vertices of which are all on the unit circle and every arrow $a$ stands on an arc of $\pi \mathrm{R}_{a}$ radians. Condition (R1) implies that such a polygon exists as the arcs add up to $2 \pi$. If $a$ and $b$ are consecutive arrows in a cycle, then one can check that the angle between the two arrows is $\frac{\pi}{2}\left(2-R_{a}-R_{b}\right)$ because it is the inscribed angle standing on the arc spanned by the rest of the cycle. Pasting all these polygons together, one gets a tiling of the plane because condition (R2) implies that the angles of the polygons at one vertex add up to $2 \pi$ (see also $[12,17])$. Such an embedding is called an embedding with isoradial cycles ${ }^{1}$.

A second ingredient we need are perfect matchings.

DEFINITION 6.3. A perfect matching is a subset of arrows $\mathcal{P} \subset \mathcal{Q}_{1}$ such that every cycle in $\mathcal{Q}_{2}$ has exactly one arrow from $\mathcal{P}$. A perfect matching gives a nonnegative grading on $A_{\mathcal{Q}}$ by assigning degree 1 to each arrow in $\mathcal{P}$ and zero to the others:

$$
\operatorname{deg}_{\mathcal{P}} a= \begin{cases}1, & a \in \mathcal{P}, \\ 0, & a \notin \mathcal{P} .\end{cases}
$$

For an embedding with isoradial cycles, we can construct special perfect matchings:

LemMa 6.4 Definition of $\mathcal{P}_{\theta}^{ \pm}$. Given a $\mathcal{Q}$ embedded with isoradial cycles and a direction $\theta$, then the set $\mathcal{P}_{\theta}^{+}\left(\mathcal{P}_{\theta}^{-}\right)$of all arrows a such that the ray from the centre of its positive cycle in direction $\theta$ in the isoradial embedding hits a but not in its head (tail) is a perfect matching.

\footnotetext{
${ }^{1}$ Such an embedding is a bit different from an isoradial embedding of the dimer model, which embeds the dual graph isoradially, i.e. the centres of the faces sharing a common vertex lie on a unit circle. Our definition also includes cases where the cycle does not contain the centre of the circle (when one of the arrows has R-charge $\geq 1$ ).
} 
Proof. It is clear from the construction that every positive cycle has exactly one arrow in $\mathcal{P}_{\theta}^{ \pm}$. The same holds for the negative cycles because $a \in \mathcal{P}_{\theta}^{ \pm}$if and only if the ray from the centre of its negative cycle in direction $-\theta$ in the isoradial embedding hits $a$ but not in its tail (head).

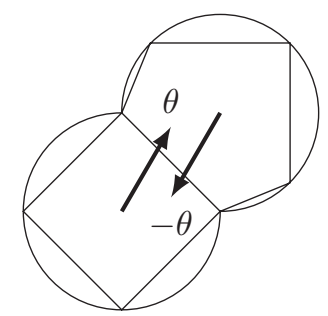

Now, let $\mathcal{Z}=\mathcal{Z}_{a}^{+}$be a zigzag path in a dimer model embedded with isoradial cycles. We define $\epsilon_{\mathcal{Z}} \in \mathbb{R} / 2 \pi \mathbb{R}$ to be the angle of $h(a)$ as viewed from the centre of the positive cycle containing $\mathcal{Z}_{a}^{+}[1] \mathcal{Z}_{a}^{+}[0]$. It is easy to check that this definition does not depend on the $a$.

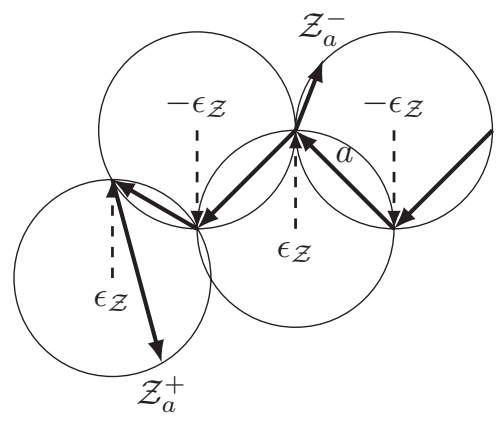

LEMMA 6.5. Let $\theta=\epsilon_{\mathcal{Z}_{a}^{ \pm}}$.

(1) $\mathcal{P}_{\theta}^{ \pm}$intersects $\mathcal{Z}_{a}^{ \pm}$in all the arrows $\mathcal{Z}_{a}^{ \pm}[i]$ with $i$ odd.

(2) Both $a$ and $\mathcal{Z}_{a}^{\mp}[1]$ are not in $\mathcal{P}_{\theta}^{ \pm}$.

Proof. We prove the statement for $\theta=\epsilon_{\mathcal{Z}_{a}^{+}}$. As illustrated above, viewed from the centres of the positive cycles, $t\left(\mathcal{Z}_{a}^{+}[i]\right)$ points in direction $\theta$ for all odd $i$. Viewed from the centre of the negative cycle, $t(a)$ points in the direction $-\theta$, so the arrow $b=\mathcal{Z}_{a}^{+}[-1]$ with head $t(a)$ must sit in $\mathcal{P}_{\theta}^{+}$, this cannot be $\mathcal{Z}_{a}^{-}[1]$ because the cycle containing $a b$ has length at least 3 .

We are now ready to prove the equivalence between cancellation and the existence of a consistent R-charge.

THEOREM 6.6. Let $\mathcal{Q}$ be a dimer model on a torus, then $\mathcal{Q}$ is cancellation if and only if it admits a consistent $\mathrm{R}$-charge.

Proof. We will prove the equivalence of the existence of a consistent R-charge with property Z. After that we can apply Theorem 5.5.

The condition is sufficient. Suppose $\mathcal{Q}$ has an R-charge and construct the corresponding tiling of the plane with isoradial cycles. Suppose $\mathcal{Z}_{a}^{+}[i]=\mathcal{Z}_{a}^{-}[j]=b$ and 
let $p_{+}$and $p_{-}$be the positively and negatively irreducible paths in the opposite direction such that $h\left(p_{+}\right)=h\left(p_{-}\right)=t(a)$ and $t\left(p_{+}\right)=t\left(p_{-}\right)=h(b)$.

If we take $\theta=\epsilon_{\mathcal{Z}_{a}^{+}}$, then it follows from Lemma 6.5 that $\operatorname{deg}_{\mathcal{P}_{\theta}^{+}} p_{+}=0$. This implies that neither $a$ nor $\mathcal{Z}_{a}^{-}[1]$ sit in $\mathcal{P}_{\theta}^{+}$. But $\mathcal{P}_{\theta}^{+}$must contain an arrow of the cycle through $\mathcal{Z}_{a}^{-}[1] a$, so $\operatorname{deg}_{\mathcal{P}_{\theta}^{+}} p_{-}>0$. This means that $p_{-}=\ell^{k} p_{+}$in $\hat{A}_{\mathcal{Q}}$ for some $k>0$, so $\mathrm{R}_{p_{-}}>\mathrm{R}_{p_{+}}$.

On the other hand, if we take $\theta=\epsilon_{\mathcal{Z}_{a}^{-}}$, then for similar reasons, $\operatorname{deg}_{\mathcal{P}_{\theta}^{-}} p_{-}=0$ but $\operatorname{deg}_{\mathcal{P}_{\theta}^{-}} p_{+}>0$ and we get in $\hat{A}_{\mathcal{Q}}, p_{+}=\ell^{l} p_{-}$for some $l>0$ and $\mathrm{R}_{p_{-}}<\mathrm{R}_{p_{+}}$. This contradicts the previous paragraph.

The condition is necessary. Every zigzag path $\mathcal{Z}$ on the torus $|\mathcal{Q}|$ is periodic, and hence its lift $|\tilde{\mathcal{Q}}|$ can be assigned a direction vector in the Euclidean plane. The unit vector in this direction will be denoted by $e_{\mathcal{Z}}$.

From condition $(\mathrm{Z})$, we can deduce that $e_{\mathcal{Z}_{a}^{+}} \neq e_{\mathcal{Z}_{a}^{-}}$for every arrow $a$. If this were not the case, $\mathcal{Z}_{a}^{+}$and $\mathcal{Z}_{a}^{-}$would intersect an infinite number of times (in shifts of $a$ in the direction $e_{\mathcal{Z}_{a}^{-}}$.

We now define an R-charge as $\frac{1}{\pi}$ times the positive angle from $e_{\mathcal{Z}_{a}^{-}}$to $e_{\mathcal{Z}_{a}^{+}}$

$$
\mathrm{R}_{a}:=\frac{1}{\pi} \measuredangle\left(e_{\mathcal{Z}_{a}^{-}}, e_{\mathcal{Z}_{a}^{+}}\right)
$$

The value of $R_{a}$ is always nonzero and smaller than 2 .

We now prove that the following two conditions hold:

$$
\sum_{a \in c} \mathrm{R}_{a}=2 \text { and } \sum_{h(a)=v}\left(1-\mathrm{R}_{a}\right)+\sum_{t(a)=v}\left(1-\mathrm{R}_{a}\right)=2 .
$$

First look at the incidence structure of the zag rays starting from arrows around a positive cycle $c$ (i.e., the $\overrightarrow{\mathcal{Z}}_{a}^{-}$). These rays do not intersect. If $a, b$ are consecutive arrows, the intersection of $\overrightarrow{\mathcal{Z}}_{a}^{-}$and $\overrightarrow{\mathcal{Z}}_{b}^{-}$would imply that $\overrightarrow{\mathcal{Z}}_{a}^{+}=a \overrightarrow{\mathcal{Z}}_{b}^{-}$and $\overrightarrow{\mathcal{Z}}_{a}^{-}$intersect twice. If $a$ and $b$ are not consecutive in the cycle, there must be an arrow $u$ between $a$ and $b$. But then, $\overrightarrow{\mathcal{Z}}_{u}^{-}$must either intersect $\overrightarrow{\mathcal{Z}}_{a}^{-}$or $\overrightarrow{\mathcal{Z}}_{b}^{-}$. Proceeding in the same way, we can always find two consecutive arrows for which the zag rays intersect.

The non-intersection implies that the directions $e_{\mathcal{Z}_{a}^{-}}$are ordered on the unit circle in the same way as the arrows $a$. For consecutive arrows $a, b e_{\mathcal{Z}_{a}^{+}}=e_{\mathcal{Z}_{b}^{-}}$so that the sum of the angles add up to $2 \pi$ and hence the sum of the R-charges is 2 .

We can now repeat this for the vertices. Look at all arrows leaving a vertex $v$. The zig rays of two consecutive leaving arrows do not intersect because otherwise we could follow the second zig path backwards inside the piece cut out by the two zig rays. This backwards path must leave this piece by an arrow $b$ of the first zig ray (because the second zag path cannot self-intersect). But now the zigzag rays from $b$ intersect twice, which contradicts $Z$. If two zag rays of nonconsecutive leaving arrows intersect, then the zig ray of an arrow in between must intersect one of these zig rays so that we can always reduce to the consecutive case.

The angle between the directions of the zig rays of two consecutive leaving arrows $a_{1}$ and $a_{2}$ is $\pi\left(2-\mathrm{R}_{a_{1}}-\mathrm{R}_{b}\right)$ where $b$ is the incoming arrow between $a_{1}$ and $a_{2}$. The fact that 
these angles add up to $2 \pi$ gives us the second consistency condition.
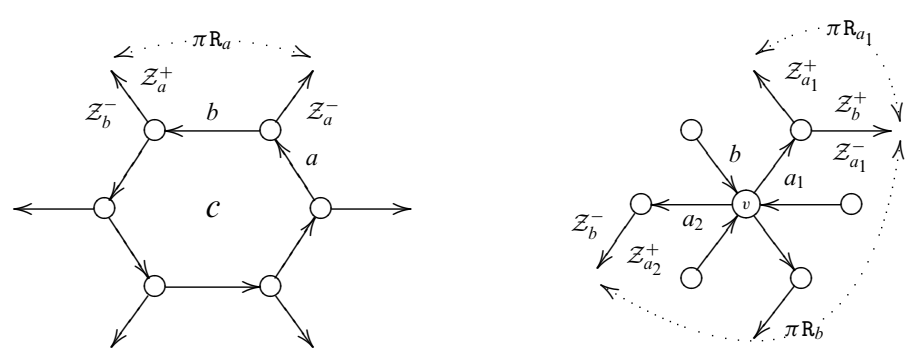

REMARK 6.7. The first part of this theorem is an extension of Lemma 5.3.1 in [10] to the marginally consistent case.

REMARK 6.8. The second part of the theorem gives us an R-charge from the directions of the zigzag paths in the plane. We can use this R-charge to construct an embedding with isoradial cycles. Note, however, that the angles between the zigzag paths in this new embedding are in general not the same as the ones we used to construct the R-charge. We can recover these original directions from the embedding with isoradial cycles, because the $e_{\mathcal{Z}_{a}^{+}}$is the original embedding point precisely in the directions $\epsilon_{\mathcal{Z}_{a}^{+}}$of the new embedding.

7. Algebraic consistency. In [5], Broomhead introduced the notion of algebraic consistency. For this, he constructed a second algebra from the dimer model: $B_{\mathcal{Q}}$. From $\mathcal{Q}$, one can construct the following diagram of maps:

$$
\mathbb{Z} \stackrel{e}{\leftarrow} \mathbb{Z}^{Q_{2}} \stackrel{d}{\rightarrow} \mathbb{Z}^{Q_{1}} \stackrel{d}{\rightarrow} \mathbb{Z}^{Q_{0}},
$$

with $e(c)=1$ and $d(c)=\sum_{a \in c} a$ for any cycle $c \in Q_{2}$ and $d(a)=h(a)-t(a)$. We define $M=\mathbb{Z}^{Q_{1}} / d e^{-1}(0)$ and for any vertices $i, j$, we set

$$
M_{i j}^{+}=\frac{d^{-1}(i-j) \cap n^{Q_{1}}}{d e^{-1}(0)} .
$$

Then the $B$-algebra is defined as

$$
B_{\mathcal{Q}}=\bigoplus_{i, j \in Q_{0}} \operatorname{Span}\left(M_{i j}^{+}\right) \subset \operatorname{Mat}_{\left|Q_{0}\right|}(\mathbb{C}[M]) .
$$

There is a natural map $\tau: A_{\mathcal{Q}} \rightarrow B_{\mathcal{Q}}: a \mapsto a \in \operatorname{Span}\left(M_{h(a) t(a)}\right)$.

DEFINITION 7.1. A dimer model is called algebraicly consistent if and only if $\tau$ : $A_{\mathcal{Q}} \rightarrow B_{\mathcal{Q}}$ is an isomorphism.

In [5], Broomhead proved:

THEOREM 7.2 Broomhead. If a dimer model admits a geometrically consistent Rcharge, then it is algebraically consistent.

In this section, we will extend this result to any consistent R-charge. The proof given will follow the same lines as Broomhead's proof. In particular, we will use the following important lemma: 
Lemma 7.3 [5, Lemma 6.1.1]. If $\mathcal{Q}$ is a dimer model on a torus, then $A_{\mathcal{Q}}$ is algebraically consistent if it is cancellation and for every pair of vertices $v$ and $w$ in the universal cover $\tilde{\mathcal{Q}}$, there is a path $p: v \rightarrow w$ and a perfect matching $\tilde{\mathcal{P}}$ that does not meet $p$.

We also need these three lemmas.

Lemma 7.4. Suppose $A_{\mathcal{Q}}$ is cancellation and $\operatorname{deg}: Q_{1} \rightarrow \mathbb{R}$ is any (not necessarily positive) grading such that $\operatorname{deg} \ell \neq 0$. Then, two paths in $A_{\mathcal{Q}}$ are equivalent if and only if they are homotopic and have the same deg-degree.

Proof. It is clear that the relations $r_{a}$ imply that equivalent paths are homotopic and must have the same degree. Because homotopies in the dimer model are generated by substituting paths $p \rightarrow q$ such that $p q^{-1}=\ell$, homotopic paths can only differ by a factor $\ell^{k}$. The degree of $\ell$ is not zero, so if homotopic paths have the same degree, they must be equal in $A_{\mathcal{Q}}$.

LEMMA 7.5. If $A_{\mathcal{Q}}$ admits a consistent $\mathrm{R}$-charge, $\mathcal{P}$ is a perfect matching and $p$ and $q$ are cyclic paths with opposite homology classes, then either $p$ or $q$ meets $\mathcal{P}$.

Proof. Suppose $\operatorname{deg}_{\mathcal{p}} p=\operatorname{deg}_{\mathcal{p}} q=0$. Take any path $r$ from $h(p)$ to $h(q)$; then $\operatorname{deg}_{\mathcal{P}}(p r q)=\operatorname{deg}_{\mathcal{P}} r$ and $p r q$ and $r$ have the same homology class, so by Lemma 7.4 , they must be the same. But this is impossible because for the R-charge, prq and $r$ must have different degrees.

LEMMA 7.6. If $\mathcal{Q}$ satisfies condition $(Z)$ and $\chi_{\mathcal{Q}}=0$, then given a zigzag path $\mathcal{Z}_{1}$ in the universal cover, there is always another zigzag path $\mathcal{Z}_{2}$ making a positive angle with $\mathcal{Z}_{1}$ less than $\pi$ radians: $0<\measuredangle\left(e_{\mathcal{Z}_{1}}, e_{\mathcal{Z}_{2}}\right)<\pi$.

Proof. Suppose that the lemma does not hold. Let $\mathcal{Z}_{2}$ be the zigzag path whose angle with $\mathcal{Z}_{1}$ is smallest and let $a$ be an arrow in their intersection. There are two possibilities: $\mathcal{Z}_{1}=\overrightarrow{\mathcal{Z}}_{a}^{+}$and $\mathcal{Z}_{2}=\mathcal{Z}_{a}^{-}$or $\mathcal{Z}_{1}=\overrightarrow{\mathcal{Z}}_{a}^{-}$and $\mathcal{Z}_{2}=\mathcal{Z}_{a}^{+}$. In the first case, the directions in the zigzag paths show that there must be another arrow in the intersection behaving like the second case.

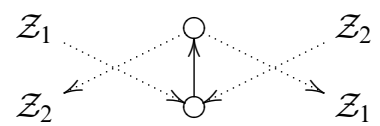

So, suppose $\mathcal{Z}_{1}=\overrightarrow{\mathcal{Z}}_{a}^{+}$and $\mathcal{Z}_{2}=\mathcal{Z}_{a}^{-}$and let $b=\mathcal{Z}_{a}^{+}[-1]$. By our hypothesis, the zigzag path $\mathcal{Z}_{b}^{+}$makes a positive angle with $\mathcal{Z}_{1}$ that is at least as big as the angle with $\mathcal{Z}_{2}$. Therefore, the $\mathcal{Z}_{b}^{+}$must intersect $\mathcal{Z}_{1}$ a second time, but by condition $(\mathrm{Z}), \mathcal{Z}_{b}^{+}[i]=$ $\mathcal{Z}_{1}[j] \Longrightarrow i j<0$. This also implies an intersection of $\mathcal{Z}_{b}^{+}$with $\mathcal{Z}_{2}$. This implies that $\mathcal{Z}_{2}$ and $\mathcal{Z}_{b}^{+}$cannot have the same direction; otherwise, they would intersect multiple times in this direction (take shifts of the intersection). So, $\mathcal{Z}_{b}^{+}$makes a positive angle with $\mathcal{Z}_{1}$ that is bigger than the angle with $\mathcal{Z}_{2}$. Now this implies a second intersection with $\mathcal{Z}_{2}$ 
contradicting $\mathrm{Z}$.

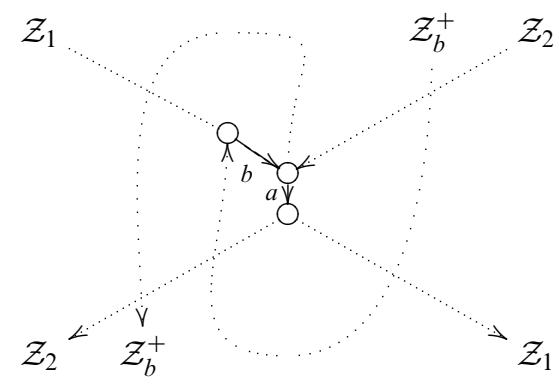

THEOREM 7.7. A dimer model with $\chi_{\mathcal{Q}}=0$ is algebraically consistent if and only if it is cancellation.

Proof. Note that algebraic consistency automatically implies that cancellation as $B_{\mathcal{Q}}$ is a subalgebra of the cancellation algebra $\operatorname{Mat}_{n}(\mathbb{C}[M])$.

Suppose that $\mathcal{Q}$ is cancellation and let $0 \geq \theta_{1}>\cdots>\theta_{u}>2 \pi$ be the directions of the zigzag paths. Use these directions to construct an R-charge as in Theorem 6.6 and its corresponding embedding with isoradial cycles. For each $i \in\{1, \ldots, u\}$, we define $\mathcal{P}_{i}:=$ $\mathcal{P}_{\theta_{i}}^{+}$(note that by the isoradial construction, $\theta_{i}=\epsilon_{\mathcal{Z}_{i}}$ ).

Every vertex in the universal cover has an arriving and a leaving arrow not in $\mathcal{P}_{i-1} \cup$ $\mathcal{P}_{i}$. Indeed if we look at the arrows in a vertex $v$, then by Remark 6.8, every arrow is a vector from $e_{\mathcal{Z}_{a}^{-}}$to $e_{\mathcal{Z}_{a}^{+}}$, so the tail of each arriving arrow $a$ and the head of the leaving arrow $b$ in the same negative cycle are both in the same direction viewed from their positive cycles. So, if we shift all arrows arriving in and leaving from $v$ to the unit circle, they will form a path as illustrated below.
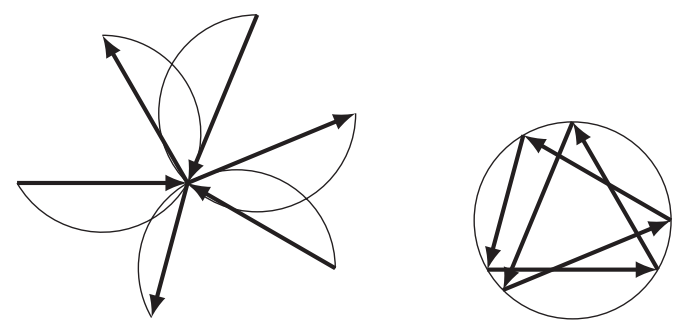

The second consistency condition implies that the path goes around the unit circle $n-1$ times where $n$ is the number of incoming arrows.

$$
\sum_{h(a)=v}\left(1-\mathrm{R}_{a}\right)+\sum_{t(a)=v}\left(1-\mathrm{R}_{a}\right)=2 \Longrightarrow \sum_{h(a)=v} \mathrm{R}_{a}+\sum_{t(a)=v} \mathrm{R}_{a}=2 n-2 .
$$

An arrow sits in $\mathcal{P}_{i-1} \cup \mathcal{P}_{i}$ if and only if its head, tail or body cross the direction $\theta_{i}$. If all incoming arrows would cross $\theta_{i}$, the path would go round $n$ times which is a contradiction. The same can be said about the leaving arrows.

This means that there is a path from every vertex $v$ that does not meet $\mathcal{P}_{i-1} \cup \mathcal{P}_{i}$, and hence does not intersects the zigzag path $\mathcal{Z}_{i}$. It also does not self-intersect because it does not meet $\mathcal{P}_{i}$. Therefore, it must either be parallel or antiparallel ${ }^{2}$. Parallel is impossible

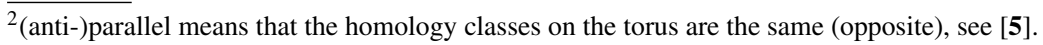


because of Lemma 7.5 and the existence of a path in the opposite direction of the zigzag path.

Let us call this ray $\mathcal{Y}_{i}^{v}$. If $\mathcal{Y}_{i}^{v}$ and $\mathcal{Y}_{i+1}^{v}$ intersect multiple times, we know that the pieces between the intersections are equivalent because they both do not meet $\mathcal{P}_{i}$. Hence, they also both do not meet $\mathcal{P}_{i \pm 1}$. We can chose $\mathcal{Y}_{i}^{v}$ and $\mathcal{Y}_{i+1}^{v}$ to overlap on that piece. Choosing the $\mathcal{Y}_{i}^{v}$ this way, we can divide the plane into sectors lying between the $\mathcal{Y}_{i}^{v}$ and $\mathcal{Y}_{i+1}^{v}$.

Now let $w$ be another vertex in the universal cover. If it lies on one of the rays $\mathcal{Y}_{i}$, then there is a path from $v$ to $w$ that does not meet $\mathcal{P}_{i}$. If it lies between $\mathcal{Y}_{i}^{v}$ and $\mathcal{Y}_{i+1}^{v}$, we can find a vertex $u_{1}$ far enough on $\mathcal{Y}_{i}^{v}$ and $u_{2}$ far enough on $\mathcal{Y}_{i+1}^{v}$ such that $w$ lies in the piece cut out by $\mathcal{Y}_{i}^{v}, \mathcal{Y}_{i+1}^{u_{1}}, \mathcal{Y}_{i}^{u_{2}}$ and $\mathcal{Y}_{i+1}^{v}$. Note that the middle two paths intersect because by Lemma 7 , the angle in the original embedding between them is smaller than $\pi$.

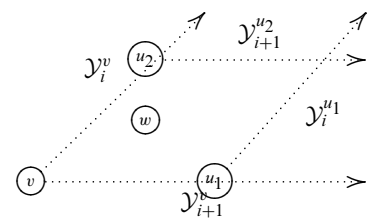

The piece is bounded by two paths that do not meet $\mathcal{P}_{i}$, so they have the same homology and $\operatorname{deg}_{\mathcal{P}_{i}}$ and by Lemma 7.4, they are equivalent. Hence, there is a sequence of relations turning the first path into the second. One of the intermediate steps must meet $w$ because it is inside the piece. This will give us a path from $v$ to $w$ that does not meet $\mathcal{P}_{i}$.

REMARK 7.8. The idea of cutting out a piece bounded by paths that do not meet a certain perfect matching is borrowed from Section 6.3.1 in [5]. In order to make this work in the marginal consistent case, we used the new notion of these $\mathcal{P}_{\theta}$ which do not appear in [5].

\section{Orders.}

DEFINITION 8.1. An order $A$ is a prime algebra (i.e. the product of nonzero ideals is nonzero) which is a finitely generated module over its centre $R$. If $K$ is the quotient field of $R$ and $\Delta=A \otimes_{R} K$, then we say that $A$ is an $R$-order in $\Delta$.

Orders have a special property: Reichstein and Vonessen [21] showed they can be reconstructed from a certain representation space. Suppose $A$ can be written as a path algebra with relations $\mathbb{C} Q / \mathcal{I}$.

For any dimension vector $\alpha$, we can $\operatorname{define} \operatorname{rep}(Q, \alpha)$ as

$$
\operatorname{rep}(Q, \alpha):=\bigoplus_{a \in Q_{1}} \operatorname{Mat}_{\alpha_{h(a)} \times \alpha_{t(a)}}(\mathbb{C}) .
$$

This space parametrizes the $\alpha$-dimensional representations of $Q$.

On this space, we have a base change action of the group $\mathrm{GL}_{\alpha}=\prod_{v \in Q_{0}} \mathrm{GL}_{\alpha_{v}}(\mathbb{C})$. This group also acts on $\operatorname{Mat}_{|\alpha|}(\mathbb{C})$ by base change and we define $\operatorname{Eqv}(Q, \alpha)$ as the ring of equivariant polynomial maps from $\operatorname{rep}(Q, \alpha)$ to $\operatorname{Mat}_{|\alpha|}(\mathbb{C})$

$$
\operatorname{Eqv}(Q, \alpha):=\left\{f: \operatorname{rep}(Q, \alpha) \rightarrow \operatorname{Mat}_{|\alpha|}(\mathbb{C}) \mid \forall g \in \mathrm{GL}_{\alpha}: f\left(\rho^{g}\right)=f(\rho)^{g}\right\}
$$

The multiplication in this ring comes from $\operatorname{Mat}_{|\alpha|}(\mathbb{C})$. 
For $A$, we define the $\operatorname{simples}(A, \alpha)$ as the subset of $\operatorname{rep}(Q, \alpha)$, containing all representations of $Q$ that are simple representation of $A$.

THEOREM 8.2 [21, Theorem 6.4]. If $A$ is an orde, then there is an $\alpha$ such that

$$
A \cong \frac{\operatorname{Eqv}(Q, \alpha)}{\{f: f(\operatorname{simples}(A, \alpha))=0\}}
$$

REMARK 8.3. The original version of the theorem uses the terminology of PI-rings which is a bit broader than orders. We also reformulated the theorem in the language of quivers, whereas the original works with generators of an algebra. The dimension vector is such that the PI-degree of $A$ is $|\alpha|$, this is the biggest $\alpha$ for which there exist simples or equivalently the dimension vector of a simple representation of the form $\rho: A \rightarrow A \otimes_{R}$ $R / \mathfrak{m}$, where $\mathfrak{m} \triangleleft R$ is a maximal ideal.

In the case of dimer models, we already had a notion of reconstructing the algebra $A$ using the algebraic consistency. Algebraic consistency fits in this more general framework because of the following lemma:

LEMMA 8.4. If $\mathcal{Q}$ is a dimer model, then $B_{\mathcal{Q}} \cong \frac{\operatorname{Eqv}(Q, \alpha)}{\left\{f: f\left(\operatorname{simples}\left(A_{\mathcal{Q}}, \alpha\right)\right)=0\right\}}$ with $\alpha$ the dimension vector that assigns 1 to each vertex.

Proof. One can easily check that using the terminology of the previous section

$$
\operatorname{Eqv}(Q, \alpha)=\bigoplus_{i, j} \operatorname{Span}\left(d^{-1}(i-j) \cap n^{Q_{1}}\right) \subset \operatorname{Mat}\left(\mathbb{C}\left[\mathbb{Z}^{Q_{1}}\right]\right)
$$

If two monomials of $x^{\alpha}, x^{\beta} \in \mathbb{C}\left[\mathbb{Z}^{Q_{1}}\right]$ evaluate the same on all simples, then they evaluate the same on all representations for which all arrows are nonzero. This implies that $\alpha-\beta \in$ $d e^{-1}(0)$ because such a simple representation is a representation of $A_{\mathcal{Q}}$ if and only if all cycles evaluate to the same number. Therefore,

$$
\frac{\operatorname{Span}\left(d^{-1}(i-j) \cap n^{Q_{1}}\right)}{\left\{f: f\left(\operatorname{simples}\left(A_{\mathcal{Q}}, \alpha\right)\right)=0\right\}}=\operatorname{Span}\left(\frac{d^{-1}(i-j) \cap n^{Q_{1}}}{d e^{-1}(0)}\right) .
$$

So, algebraic consistency seems just a specific consequence of being an order and indeed we have the following theorem:

THEOREM 8.5. A Jacobi algebra of a dimer model on a torus is an order if and only if it is algebraically consistent.

Proof. An algebraically consistent dimer model is always an order because $B_{\mathcal{Q}}$ by construction is prime and finite over its centre. If $A_{\mathcal{Q}}$ is an order, then it is cancellation because it is prime. Theorem 7.7 implies that it is algebraically consistent.

REMARK 8.6. The Jacobi algebra of a dimer model on a higher genus surface can never be an order. Indeed if the algebra is prime, then the central element $\ell$ cannot be a zero divisor, so $A_{\mathcal{Q}}$ embeds in $\hat{A}_{\mathcal{Q}}=A_{\mathcal{Q}} \otimes \mathbb{C}\left[\ell, \ell^{-1}\right]$ and the latter must also be prime and finite over its centre. From [2, Theorem 8.4], we know that $\hat{A}_{\mathcal{Q}}$ is the matrix algebra of a fundamental group algebra of a three-dimensional manifold. By construction, this 
manifold is a circle bundle over a higher genus surface group. Such algebras are never finite over their centres.

A dimer model on a sphere is an order if and only if it is cancellation. Indeed if it is cancellation and we are on a sphere, then every path $p$ is isomorphic to a $\ell^{k} p^{\prime}$, where $p^{\prime}$ does not self-intersect. There are only a finite number of paths that do not self-intersect, so $A_{\mathcal{Q}}$ is finite over the central subalgebra $\mathbb{C}[\ell]$. On the other hand, if $A_{\mathcal{Q}}$ is an order, then it is cancellation because it is prime. Because we have a simple representation $A_{\mathcal{Q}} \rightarrow$ $A_{\mathcal{Q}} \otimes_{\mathbb{C}[\ell]} \mathbb{C}[\ell] /(\ell-1)=\operatorname{Mat}_{\left|Q_{0}\right|}(\mathbb{C})$, the $\alpha$ from Theorem 8.2 and its consequent remark must be the dimension vector that assigns 1 to each vertex. Lemma 8.4 and Theorem 8.2 imply that the algebra is algebraically consistent.

9. Noncommutative crepant resolutions. In [23], Van den Bergh introduced the notion of a noncommutative crepant resolution.

DEFINITION 9.1. Let $R$ be an affine commutative Gorenstein domain, with quotient field $K$. An algebra $A$ is a noncommutative crepant resolution of $R$ if $A$ is homologically homogeneous (i.e. the projective dimension of all simple representations of $A$ is the same) and $A \cong \operatorname{End}_{R}(M)$ for some finitely generated reflexive $R$-module $M$ (reflexive means $\left.\operatorname{Hom}_{R}\left(\operatorname{Hom}_{R}(M, R), R\right) \cong M\right)$.

As is explained in the discussion following this definition in and using results [23] from [18] and [20], this definition is satisfied if

(1) $A$ is an $R$-order in $\operatorname{Mat}_{n \times n}(K)$,

(2) $A$ has finite global dimension,

(3) $A$ is Cohen-Macaulay as an $R$-module,

(4) the ramification locus has codimension $\geq 2$.

The ramification locus of an order is defined as the set of points $\mathfrak{p} \in \operatorname{Mspec} R$ such that $A \otimes_{R} R / \mathfrak{p} \neq \operatorname{Mat}_{n \times n}(\mathbb{C})$ (or in other words, the representation of $A$ at the point $p$ is not simple).

THEOREM 9.2. The Jacobi algebra of a dimer model on a torus is a noncommutative crepant resolution of its centre if and only if it is cancellation.

Proof. If $A_{\mathcal{Q}}$ is a noncommutative crepant resolution of its centre, then it is an order and hence cancellation.

Suppose that $A_{\mathcal{Q}}$ is cancellation, then $A_{\mathcal{Q}}$ is an order. Because $A_{\mathcal{Q}} \otimes_{\mathbb{C}[\ell]} \mathbb{C}\left[\ell, \ell^{-1}\right]=$ $\operatorname{Mat}_{n}\left(\mathbb{C}\left[X_{1}^{ \pm 1}, X_{2}^{ \pm 1}, X_{3}^{ \pm 1}\right]\right)$ and $K=\mathbb{C}\left(X_{1}, X_{2}, X_{3}\right)$, we have that $A_{\mathcal{Q}} \otimes_{R} K=\operatorname{Mat}_{n \times n}(K)$. By the CY-3 property, the global dimension is 3. From [22, Theorem 2.2]. we conclude that $A$ is Cohen-Macaulay.

Finally, if we show that the ramification locus has codimension at least 2, we are done. If $\mathfrak{p} \triangleleft Z(A)$ lies in the ramification locus, then $\ell$ must be in $\mathfrak{p}$, because otherwise all arrows must evaluate to something nonzero and the representation is simple.

Now we show that there is at least one cycle with nonzero homology class that evaluates to zero: if this were not the case, we could find two cycles $c_{1}$ and $c_{2}$ with linearly independent homotopy classes that are not zero. Both cycles can be seen as $h\left(c_{1}\right) \operatorname{Tr} c_{1}$ and $h\left(c_{2}\right) \operatorname{Tr} c_{2}$ where $\operatorname{Tr} c_{1}, \operatorname{Tr} c_{2} \in R$. If $v_{1}$ and $v_{2}$ are two vertices, then we can look at $v_{1} \operatorname{Tr} c_{1}$ and $v_{2} \operatorname{Tr} c_{2}$. These are two cycles, they are nonzero and because the homotopy classes are linearly independent, they must intersect. This means that there is a path of nonzero arrows between $v_{1}$ and $v_{2}$. As this holds for every couple of vertices, the representation must be simple. 
Two zero cycles ( $\ell$ and the one with nontrivial homology) with different homology generate an ideal which defines a subscheme of codimension 2.

REMARK 9.3. A different proof of this statement can be found in [5].

REMARK 9.4. A dimer model on a higher genus surface can never be an NCCR because it is not an order. A dimer model on a sphere can never be an NCCR because even if it were an order, its centre is $\mathbb{C}[\ell]$, which is smooth so that the NCCR should be equal to $\mathbb{C}[\ell]$.

10. Summary. The following theorem is a summary of all main theorems from the previous sections:

THEOREM 10.1. For a dimer model $\mathcal{Q}$ on a torus, the following are equivalent:

(1) $A_{\mathcal{Q}}$ is cancellation.

(2) $A_{\mathcal{Q}}$ is algebraically consistent.

(3) $A_{\mathcal{Q}}$ is an order.

(4) $A_{\mathcal{Q}}$ is an NCCR of its centre.

(5) The zig and zag rays in the universal cover do not intersect twice.

(6) There exists a consistent R-charge.

The remarks following these proofs show that in the higher genus case, this theorem changes to:

THEOREM 10.2. For a dimer model $\mathcal{Q}$ on a higher genus surface, the following are equivalent:

(1) $A_{\mathcal{Q}}$ is cancellation.

(2) The zig and zag rays in the universal cover do not intersect twice.

While the following can never happen

(1) There exists a consistent R-charge.

(2) $A_{\mathcal{Q}}$ is algebraically consistent.

(3) $A_{\mathcal{Q}}$ is an order.

(4) $A_{\mathcal{Q}}$ is an NCCR of its centre.

In the genus zero case, we have got:

THEOREM 10.3. For a dimer model $\mathcal{Q}$ on a sphere, the following are equivalent:

(1) $A_{\mathcal{Q}}$ is cancellation.

(2) $A_{\mathcal{Q}}$ is algebraically consistent.

(3) $A_{\mathcal{Q}}$ is an order.

While the following can never happen:

(1) $A_{\mathcal{Q}}$ is $C Y$-3.

(2) The zig and zag rays in the universal cover do not intersect twice.

(3) There exists a consistent R-charge.

(4) $A_{\mathcal{Q}}$ is an NCCR of its centre.

ACKNOwledgements. I want to thank Nathan Broomhead, Alastair King, Michael Wemyss, Travis Schedler and Sergei Mozgovoy for the interesting discussions about these subjects at the Liegrits workshop in Oxford in January 2008 and during my stays in Bath, Chicago and Wuppertal. I also want to thank Lieven Le Bruyn for sharing his insights in the theory of reflexive Azumaya algebras. 


\section{REFERENCES}

1. R. Bocklandt, Graded Calabi Yau algebras of dimension 3, J. Pure Appl. Algebra. 212(1) (2008), 14-32.

2. R. Bocklandt, Calabi-Yau algebras and quiver polyhedra, arXiv: 0905.0232.

3. R. Bocklandt, Note on infinite CY-3 dimer models that are not cancellation.

4. T. Bridgeland, Flops and derived categories, Invent. Math. 147 (2002), 613-632.

5. N. Broomhead, Dimer models and Calabi-Yau algebras, arXiv:0901.4662. $323-362$.

6. M. C. R. Butler and A. D. King, Minimal resolutions of algebras, J. Algebra 212(1) (1999),

7. B. Davison, Consistency conditions for brane tilings, arXiv: 0812.4185.

8. S. Franco, A. Hanany, K. D. Kennaway, D. Vegh and B. Wecht, Brane dimers and quiver gauge theories, JHEP 0601 (2006), 096, hep-th/ 0504110.

9. V. Ginzburg, Calabi-Yau algebras, math/0612139.

10. A. Hanany, C. P. Herzog and D. Vegh, Brane tilings and exceptional collections, J. High Energy Phys. 7 (2006), 44.

11. A. Hanany and K. D. Kennaway, Dimer models and toric diagrams, hep-th/0602041.

12. A. Hanany and D. Vegh, Quivers, tilings, branes and rhombi, J. High Energy Phys. 10 (2007), 35 .

13. A. Ishii and $\mathrm{K}$. Ueda, On moduli spaces of quiver representations associated with brane tilings, in higher dimensional algebraic varieties and vector bundles, (RIMS Kroku Bessatsu, Kyoto, 2008).

14. A. Ishii and $\mathrm{K}$. Ueda, $A$ note on consistency conditions on dimer models, arXiv: 1012.5449.

15. K. D. Kennaway, Brane tilings, Int. J. Modern Phys. A 22(18), (2007), 29773038. hep-th/0710.1660.

16. R. Kenyon, An introduction to the dimer model (School and Conference on Probability Theory, Abdus Salam Int. Cent. Theoret. Phys., Trieste, 2004).

17. R. Kenyon and J. M. Schlenker, Rhombic embeddings of planar quad-graphs, Trans. AMS. 357(9) (2004), 3443-3458.

18. L. Le Bruyn, A cohomological interpretation of the reflexive Brauer group, J. Algebra 105 (1987), 250-254.

19. S. Mozgovoy and M. Reineke, On the noncommutative Donaldson-Thomas invariants arising from brane tilings, Adv. Math. 223(5) (2010), 1521-1544.

20. M. Orzech, Brauer Groups and Class Groups for a Krull Domain, in Brauer groups in ring theory and algebraic geometry (Springer, 1982), 68-87.

21. Z. Reichstein and N. Vonessen, Polynomial identity rings as rings of functions. J. Algebra, 310(2) (2007), 624-647.

22. J. T. Stafford and M. Van den Bergh, Non-commutative resolutions and rational singularities, Michigan Math. J. 57 (2008), 659-674.

23. M. Van den Bergh, Non-commutative crepant resolutions, in The legacy of Niels Hendrik Abel (Springer, 2002), 749-770. 